\title{
The thermal bath corrections to the Cooper pair potential
}

\author{
Miroslav Pardy \\ Department of Physical Electronics \\ Masaryk University \\ Kotlářská 2, 61137 Brno, Czech Republic \\ email:pamir@physics.muni.cz
}

July 27, 2020

\begin{abstract}
The Coulomb and Yukawa potentials of particles are derived from the Schwinger field theory at zero and the finite temperature. At the same time the correction to the Coulomb potential is determined for the two charges interacting in the photon black-body sea by the photon exchange mechanism. The running coupling constant is determined in dependence on the mean free path of photons in the photon sea. The relation to the Cooper pairs and phonon-phonon interaction at the thermal bath is discussed.
\end{abstract}

\section{Introduction}

All properties of a superconductor, including ground-state energy, critical temperature, quasi-particle energy spectra containing gaps, supercondensate density, specific heat, and supercurrent density can be computed in BCS theory without mathematical complexities.

The attraction of two electrons in BCS can be in simplicity explained. An electron in a metal normally behaves as a free particle. The electron is repelled from other electrons due to their negative charge, but it also attracts the positive ions that make up the rigid lattice of the metal. This attraction distorts the ion lattice, moving the ions slightly toward the electron, increasing the positive charge density of the lattice in the vicinity. This positive charge can attract other electrons. At long distances, this attraction between electrons due to the displaced ions can overcome the electron repulsion due to their negative charge, and cause them to pair up. The quantum theory explanation shows that the effect is due to electron-phonon interactions, with the phonon being the collective motion of the positively-charged lattice. The energy of the pairing interaction is quite weak, and thermal energy can easily break the pairs. So only at low temperatures, in metal and other substrates, are a significant number of the so called Cooper pairs. 
The electrons in a pair are not necessarily close together and paired electrons may still be many hundreds of nanometers apart. This distance is usually greater than the average inter-electron distance, so, many Cooper pairs can occupy the same space. Electrons are fermions with one half spin, but the total spin of a Cooper pair is integer, zero, or one. So, it means a Cooper pair is a composite boson. This means the wave functions are symmetric under particle interchange. Therefore, unlike electrons, multiple Cooper pairs are allowed to be in the same quantum state, which is responsible for the phenomena of superconductivity.

The determination of potential at finite temperature is one of the problems which form the basic ingredients of the quantum field theory (QFT) at finite temperature. This theory was formulated some years ago by Dolan and Jackiw (1974), Weinberg (1974) and Bernard (1974) and some of the first applications of this theory were the calculations of the temperature behaviour of the effective potential in the Higgs sector of the standard model.

Information on the systematic examination of the finite temperature effects in quantum electrodynamics (QED) at one-loop order was given by Donoghue, Holstein and Robinett (1985). They have treated the calculation of mass, charge, wave function renormalization and so on and demonstrated the running of the coupling constant at finite temperature and discussed the normalized vertex function and the energy momentum tensor.

A similar discussion of QED was published by Johansson, Peressutti and Skagerstam (1986) and Cox et. al. (1984).

The finite-temperature QED, QFT and also quantum chromodynamics usually deal with the specific processes of these theories in the heat bath of photons or other particles.

The term "finite-temperature" implies the presence of the medium formed usually by the electron-positron and photon gases which is in the thermodynamical equilibrium of some temperature $T \neq 0$. Such a definition is used, for instance, by Zhukovskii et al. (1995) in the determination of the electron mass operator of the electron at finite temperature. It is evident that the heat bath can be formed by different kinds of elementary particles and so such different hot media have a different influence on the same specific physical process developing in the media.

We use here the finite-temperature methods and Schwinger theory for determination of the Coulomb and Yukawa potentials at finite temperature. At the same time we derive the modified Coulomb potential from the two-charge interaction by the exchange photon mechanism in the photon sea (Pardy, 1994a). The phonon sea as the analogue to the photon sea is discussed.

\section{Formulation of the problem in field theory}

The basic formula of the Schwinger field theory is the vacuum-vacuum amplitude (Schwinger, 1970): 


$$
\left\langle 0_{+} \mid 0_{-}\right\rangle=e^{\frac{i}{\hbar} W}
$$

where in the case of the electromagnetic field the action $W$ is defined as (Schwinger et al., 1976)

$$
W=\frac{1}{2 c^{2}} \int(d x)\left(d x^{\prime}\right) J_{\mu}(x) D_{+}^{\mu \nu}\left(x-x^{\prime}\right) J_{\nu}\left(x^{\prime}\right),
$$

where $J^{\mu} \equiv(c \varrho, \mathbf{J})$ is the conserved current and $D_{+}^{\mu \nu}\left(x-x^{\prime}\right)$ is the photon propagator in a medium with the index of refraction $n$, the magnetic permeability $\mu$ and the dielectric constant $\varepsilon$.

The equations corresponding to the action (2) are as follows:

$$
\begin{gathered}
\Delta \varphi-\frac{\mu \varepsilon}{c^{2}} \frac{\partial^{2} \varphi}{\partial t^{2}}=-\frac{1}{\varepsilon} \varrho \\
\Delta \mathbf{A}-\frac{\mu \varepsilon}{c^{2}} \frac{\partial^{2} \mathbf{A}}{\partial t^{2}}=-\frac{\mu}{c} \mathbf{J} .
\end{gathered}
$$

The explicit form of $D_{+}^{\mu \nu}\left(x-x^{\prime}\right)$ has been obtained by as (Schwinger et al., 1976)

$$
D_{+}^{\mu \nu}=\frac{\mu}{c}\left[g^{\mu \nu}+\left(1-n^{-2}\right) \beta^{\mu} \beta^{\nu}\right] D_{+}\left(x-x^{\prime}\right),
$$

where $\beta^{\mu}=(1,0)$ and

$$
D_{+}\left(x-x^{\prime}\right)=\int \frac{(d k)}{(2 \pi)^{4}} e^{i k\left(x-x^{\prime}\right)} \frac{1}{\left|\mathbf{k}^{2}\right|-n^{2}\left(k^{0}\right)^{2}-i \epsilon}
$$

with $k^{\mu}=\left(k^{0}, \mathbf{k}\right)$. The Green function $D_{+}\left(x-x^{\prime}\right)$ can be further specified as

$$
D_{+}\left(x-x^{\prime}\right)=\frac{i}{c} \frac{1}{4 \pi^{2}} \int_{0}^{\infty} d \omega \frac{\sin \frac{n \omega}{c}\left|\mathbf{x}-\mathbf{x}^{\prime}\right|}{\left|\mathbf{x}-\mathbf{x}^{\prime}\right|} e^{-i \omega\left|t-t^{\prime}\right|}
$$

by the standard contour integral method (Schwinger et al., 1976) .

The vacuum persistence probability follows from eq. (1) in the form (Schwinger et al., 1976) :

$$
\left|\left\langle 0_{+} \mid 0_{-}\right\rangle\right|^{2}=e^{-\frac{2}{\hbar} \operatorname{Im} W}
$$

In order to derive the Coulomb law at zero temperature from the action $W$, we consider the physical situation where the photon source $J_{\mu}$ varies very slowly in time. Then, in order to obtain this limit, we write for the action (2) (Schwinger, 1970):

$$
W=\frac{1}{2 c^{2}} \int(d \mathbf{x})\left(d \mathbf{x}^{\prime}\right) d x^{0} d \tau J_{\mu}\left(\mathbf{x}, x^{0}+\tau / 2\right) D_{+}^{\mu \nu}\left(\mathbf{x}-\mathbf{x}^{\prime}, \tau\right) J_{\nu}\left(\mathbf{x}^{\prime}, x^{0}-\tau / 2\right),
$$

where $D_{+}$is given by eq. (7) with $x^{0}=c t$.

Now, let us consider the quasistationary arrangement (Schwinger, 1970) in a such sense that we begin with the vacuum, we turn on these sources slowly and then keep 
them constant for a long time $\mathrm{T}$. The fact that the sources do not change appreciably in time means that their Fourier transforms are essentially restricted to zero frequency.

The structure of $W$ then reduces to an account of the instantaneous coupling of sources involving the spatial function

$$
\begin{gathered}
\int_{-\infty}^{\infty} d \tau D_{+}\left(\mathbf{x}-\mathbf{x}^{\prime}, \tau\right)= \\
\frac{i}{c} \frac{1}{4 \pi^{2}} \int_{0}^{\infty} d \omega \frac{\sin \frac{n \omega}{c}\left|\mathbf{x}-\mathbf{x}^{\prime}\right|}{\left|\mathbf{x}-\mathbf{x}^{\prime}\right|} \int_{-\infty}^{\infty} d \tau e^{-i \frac{\omega}{c}|\tau|}=\frac{1}{4 \pi} \frac{1}{\left|\mathbf{x}-\mathbf{x}^{\prime}\right|}
\end{gathered}
$$

where we have used the relation

$$
\int_{-\infty}^{\infty} d \tau e^{-i \frac{\omega}{c}|\tau|}=\frac{2 c}{i \omega}
$$

The vacuum amplitude (1) can be written in this case in the form

$$
\left\langle 0_{+} \mid 0_{-}\right\rangle=\exp \left[-i \int d x^{0} E\left(x^{0}\right)\right]
$$

where $(c=n=\mu=1)$

$$
E\left(x^{0}\right)=\frac{1}{4 \pi} \frac{1}{2} \int d \mathbf{x} d \mathbf{x}^{\prime} \frac{\left[\varrho(\mathbf{x}, t) \varrho\left(\mathbf{x}^{\prime}, t^{\prime}\right)-\mathbf{J}(\mathbf{x}, t) \cdot \mathbf{J}\left(\mathbf{x}^{\prime}, t^{\prime}\right)\right]}{\left|\mathbf{x}-\mathbf{x}^{\prime}\right|} .
$$

Now, if we restrict the situation to the stationary regime, then we get:

$$
E=\frac{1}{4 \pi} \frac{1}{2} \int d \mathbf{x} d \mathbf{x}^{\prime} \frac{\left[\varrho(\mathbf{x}) \varrho\left(\mathbf{x}^{\prime}\right)-\mathbf{J}(\mathbf{x}) \cdot \mathbf{J}\left(\mathbf{x}^{\prime}\right)\right]}{\left|\mathbf{x}-\mathbf{x}^{\prime}\right|}
$$

The last formula implies the Coulomb and Amperian interactions of charges and closed currents. We can see that a substantial step in the derivation of the Coulomb potential was the $\tau$-integration of the Green function (7).

\section{The Coulomb potential at finite temperature}

The situation at finite temperature is similar, the difference being that the Green function $D_{+}$has the finite-temperature correction as follows (Pardy, 1989):

$$
D=D_{+}+D_{T}
$$

where

$$
D_{T}\left(x-x^{\prime}\right)=\frac{i}{c} \frac{1}{2 \pi^{2}} \int_{0}^{\infty} d \omega \frac{\sin \frac{n \omega}{c}\left|\mathbf{x}-\mathbf{x}^{\prime}\right|}{\left|\mathbf{x}-\mathbf{x}^{\prime}\right|} \frac{\cos \omega\left(t-t^{\prime}\right)}{\exp (\hbar \omega / k T)-1},
$$

where $k$ is here the Boltzmann constant.

The finite-temperature correction to the original Coulomb potential is obviously an analogue of eq. (10): 


$$
V_{T}=\int_{-\infty}^{\infty} d \tau D_{T}\left(\mathbf{x}-\mathbf{x}^{\prime}, \tau\right)
$$

Using the definition of the delta-function

$$
\delta(\omega)=\frac{2}{c \pi} \int_{-\infty}^{\infty} d \tau \cos \frac{\omega}{c} \tau
$$

and after some integration we get the final formula

$$
V_{T}=\frac{i n}{8 \pi \hbar c} k T
$$

where $n$ is the index of refraction which can be determined by the plasma fluctuation methods and it depends on temperature.

The derivation of the potential at finite temperature was performed as an analogue of the derivation of the Cerenkov radiation at finite temperature where the substantial temperature effect was involved in the finite-temperature part of the photon Green function (Pardy, 1989).

The fact that the finite-temperature Green function generates the complex potential has no classical analogue, and it is a result only of the quantum field theory. We can easily see that the classical definition of potential cannot generate the complex part. If we have a charged particle immersed in the heat bath, and if $\mathbf{F}_{\text {ext }}$ is a classical external force acting on this particle, then the average average potential corresponding to this force and to the stochastical force generated by collisions of the test particle with the particles of the heat bath is as follows:

$$
-\langle V\rangle=\int_{\mathbf{a}}^{\mathbf{b}} \mathbf{F}_{e x t} \cdot d \mathbf{x}+\int_{\mathbf{a}}^{\mathbf{b}}\left\langle\mathbf{F}_{s}\right\rangle \cdot d \mathbf{x}=\int_{\mathbf{a}}^{\mathbf{b}} \mathbf{F}_{e x t} \cdot d \mathbf{x}
$$

where $\mathbf{a}$ and $\mathbf{b}$ are space points; the potential is considered between them, and $\mathbf{F}_{s}$ is the stochastic force which is caused by the collisions of a test particle with the particles of the heat bath. The average value of this stochastic force is evidently zero. In such a way, the stochastic collisions of a test particle with the particles of the medium does not generate the complex potential in classical physics. Such potential is a result only of the quantum field theory.

The physical meaning of the imaginary part of this potential can be seen from the relation $\left|\left\langle 0_{+} \mid 0_{-}\right\rangle\right|^{2} \leq 1$, which contains an exponential term dependent on temperature and which can be interpreted as the non-stability of the vacuum-to-vacuum amplitude, or, in other words, the finite life-time of the vacuum-to-vacuum transition.

\section{The Yukawa potential}

While we have worked with the massless photon field in the previous section, now let us first determine the potential for a field of massive particles. For spin 0 we have (Schwinger, 1970) 


$$
W=\frac{1}{2} \int(d x)\left(d x^{\prime}\right) K(x) \Delta_{+}\left(x-x^{\prime}\right) K(x)
$$

and for spin 1 it is (Schwinger, 1970):

$$
\begin{gathered}
W=\frac{1}{2} \int(d x)\left(d x^{\prime}\right) \times \\
{\left[J^{\mu}(x) \Delta_{+}\left(x-x^{\prime}\right) J_{\mu}\left(x^{\prime}\right)+\frac{1}{m^{2}} \partial_{\mu} J^{\mu}(x) \Delta_{+}\left(x-x^{\prime}\right) \partial_{\nu}^{\prime} J^{\nu}\left(x^{\prime}\right)\right]}
\end{gathered}
$$

with $(c=1)$,

$$
\Delta_{+}\left(x-x^{\prime}\right)=\int \frac{(d k)}{(2 \pi)^{4}} e^{i k\left(x-x^{\prime}\right)} \frac{1}{\left|\mathbf{k}^{2}\right|-\left(k^{0}\right)^{2}+m^{2}-i \epsilon},
$$

where the last formula is the Green function for the massive field with mass $m$. For higher spin the action $W$ is more complicated.

The algorithm of the calculation of the potential corresponding to the massive field with spin is the same as for the massless field. In such a way we write

$$
V(|\mathbf{x}|)=\int d \tau \Delta_{+}(\mathbf{x}, \tau)=\frac{e^{-m|\mathbf{x}|}}{4 \pi|\mathbf{x}|}
$$

In case of the finite temperature, we use the transformation

$$
\frac{1}{p^{2}+m^{2}-i \epsilon} \longrightarrow \frac{1}{p^{2}+m^{2}-i \epsilon}+\frac{2 \pi i}{\exp \beta(E-\mu)-1} \delta\left(p^{2}+m^{2}\right),
$$

where we have generalized the Einstein-Bose distribution by adding to the energy $E$ the chemical potential $\mu$. While for photons the chemical potential can be shown to be zero, for massive particles it is necessary to use the non-zero chemical potential. This potential enters the first law of thermodynamics for cases involving a varying number of particles:

$$
d E=T d S-P d V-\mu d N
$$

where $E$ is the internal energy of a system, $S$ its entropy, $P$ is pressure and $N$ is the number of particles. For the zero value of the chemical potential the formula (25) has an infra-red divergence and must be investigated by special mathematical techniques (Weldon, 1994).

The finite-temperature part of the potential can again be obtained by the $\tau$-integration of the temperature part of (25). After the $\tau$-integration and $p^{0}$-integration, we get the formula which contains the following integral

$$
I=\int_{0}^{\infty} \frac{d p^{2}}{\sqrt{p^{2}}} \sin \left(\sqrt{p^{2}}|\mathbf{x}|\right) \delta\left(p^{2}+m^{2}\right), \quad p=|\mathbf{p}|,
$$

which is evidently equal to zero because of the properties of the $\delta$-function. 
What is suprising is that the potential is equal to zero, which means that in the case of the massive particles which generate the Yukawa potential the vacuum-vacuum amplitude is not influenced by temperature.

It is necessary to say that the derived potential is not the screening potential which is discussed in QCD (Baier; Kalashnikov, 1994) and which follows from the linear response theory (Kajantie; Kapusta, 1985).

\section{The photon propagator with radiative corrections and the corresponding potential}

First, we remember how the Coulomb potential is modified if we involve radiation corrections in the photon propagator. Then, we apply, in analogy with previous chapters, the $\tau$-integration to the temperature Green function of the photon with radiative corrections. We take here the radiative corrections expressed by the vacuum polarization diagram. According to (Dittrich, 1978; Schwinger, 1973; Pardy, 1994a; 1994b; 1994c), the photon propagator with such radiative corrections is in the momentum representation of the form:

$$
\tilde{D}(k)=D(k)+\delta D(k)
$$

or,

$$
\begin{gathered}
\tilde{D}(k)=\frac{1}{|\mathbf{k}|^{2}-\left(k^{0}\right)^{2}-i \epsilon}+ \\
+\int_{4 m^{2}}^{\infty} d M^{2} \frac{a\left(M^{2}\right)}{|\mathbf{k}|^{2}-\left(k^{0}\right)^{2}+M^{2}-i \epsilon},
\end{gathered}
$$

the last term in equation (29) being derived on the virtual photon condition

$$
|\mathbf{k}|^{2}-\left(k^{0}\right)^{2}=-M^{2},
$$

where, for simplicity, we consider the index of refraction of the medium equal to 1 . The weight function $a\left(M^{2}\right)$ has been derived in the following form (Dittrich, 1978; Schwinger, 1973; Pardy, 1994a; 1994b; 1994c):

$$
a\left(M^{2}\right)=\frac{\alpha}{3 \pi} \frac{1}{M^{2}}\left(1+\frac{2 m^{2}}{M^{2}}\right)\left(1-\frac{4 m^{2}}{M^{2}}\right)^{1 / 2} .
$$

The x-representation of $D(k)$ in eq. (1) is given by the Fourier transform:

$$
\tilde{D}_{+}\left(x-x^{\prime}\right)=\int \frac{(d k)}{(2 \pi)^{4}} e^{i k\left(x-x^{\prime}\right)} \tilde{D}(k)
$$

or, 


$$
\tilde{D}_{+}\left(x-x^{\prime}\right)=D_{+}\left(x-x^{\prime}\right)+\int_{(2 m)^{2}}^{\infty} d M^{2} a\left(M^{2}\right) \Delta_{+}\left(x-x^{\prime}, M^{2}\right) .
$$

The introduction of the modified photon propagation function implies a change of interaction between static charges, i.e. the change of the Coulomb potential. By the same procedure as in the preceding text, we get $(c=1)$ :

$$
\begin{gathered}
\tilde{V}(|\mathbf{x}|)=\int_{-\infty}^{\infty} d \tau \tilde{D}_{+}(x)=\int_{-\infty}^{\infty} d \tau \int \frac{(d k)}{(2 \pi)^{4}} \tilde{D}(k) e^{i k x}= \\
\frac{1}{4 \pi|\mathbf{x}|}+\frac{\alpha}{3 \pi} \int_{(2 m)^{2}}^{\infty} \frac{d M^{2}}{M^{2}}\left(1+\frac{2 m^{2}}{M^{2}}\right)\left(1-\frac{4 m^{2}}{M^{2}}\right)^{1 / 2} \frac{e^{-M|\mathbf{x}|}}{4 \pi|\mathbf{x}|}
\end{gathered}
$$

In the case of the finite temperature regime, we use eqs. (18) and (25) in order to get $(c=\hbar=1)$ :

$$
V_{T}(|\mathbf{x}|)=\frac{i}{8 \pi} k T
$$

In other words, the temperature has no influence on forming the additional potential corresponding to radiative corrections.

The probability of the persistence of the vacuum follows from the vacuum amplitude (9) in the form (6):

$$
\left|\left\langle 0_{+} \mid 0_{-}\right\rangle\right|^{2}=e^{-\frac{2}{\hbar} \operatorname{Im} W},
$$

where in the case of the finite temperature situation $\operatorname{Im} W$ in general contains the imaginary part of the potential. Physically it means that the vacuum-vacuum transition of the physical system is influenced by the temperature environment. Since the radiative corrections term in eq. (33) involves the massive Green function, which at zero temperature gives Yukawa behaviour of the potential and at non-zero temperature is equal to zero, we can observe in experiment only contributions from the zero-temperature radiative corrections with the decay factor following from eq. (36).

\section{The two-body interaction in the photon sea with photon-photon interaction}

In the previous chapters we supposed that the potential of the particles is initiated by the massless or massive fields of these particles. In order to get the zero temperature and finite temperature potentials we used the formal derivation by means of the Green functions. Now, we consider the situation where the heat bath is the medium of photons with the temperature $T$ and the exchange photon can interact with the photons of the heat bath. The physical realization of such a medium is, for instance, the photons in the internal space of the black body with temperature $T$. The problem which we solve is one of the problems of QED in the photon sea and has its origin in the article by Tryon (1974). However, 
while Tryon in his article considers only the photon-electron interaction, we will consider here especially the photon-photon interaction where the exchanged photon interacts with thermal bath photons. We use here the notion of the mean free paths of photons in order to get the corrections to the two-body potential in the heat bath of photons. We use such an approach here because the considered processes can be visualized by Feynman diagrams and because quantum field theory does not immediately give a solution to this problem.

It has been shown many times that the heat bath of photons which forms the thermodynamical medium moving stochastically has an influence on the mass of the charged particles in that medium. On the other hand, the photon-photon interaction has an immediate influence on the behaviour of the interaction potential, and has therefore an influence on the coupling constant, in accordance with the general idea that the coupling constant depends on temperature.

Considering the thermal bath of photons inside the black body, it is evident that there are two fundamental situations of the interaction of the two charges in the photon sea.

The first situation is the process where there is the photon exchange between two charges on the condition that the exchanged photons are not influenced by the photon sea. It means that the thermal photons do not interact with the exchanged photons. According to QED this process corresponds to the Coulomb potential $V$.

The second situation is the process where the exchanged photons interact with the photon of the black-body photon sea. In other words, the interacting of the two charges is realized through the photon-photon interaction.

While the the first process is of second order in the fine structure constant $\alpha$, as easily follows from the corresponding Feynman diagrams, the second process is of sixth order and it involves the scattering of light by light where the ingoing and outgoing particles are the black-body photon and the exchanged photon.

Since the cross-section of the photon-photon interaction is vera small, it can be neglected in comparison with the electron-electron scattering.

In such a way the situation of the two-body interaction consists photon exchange between charges which can be destroyed by the photon sea. According to Partovi (1994) the collision frequency $\nu_{\gamma \gamma}$ of a given photon can be expressed by the relation:

$$
\nu_{\gamma \gamma}(k)=\frac{4448 \pi^{3} \alpha^{2}}{455625} c r_{e}^{2} k^{3}\left(T / T_{e}\right)^{6},
$$

where $k$ is a photon momentum, $r_{e}$ is the classical electron radius and $T_{e}=m=5.9 \mathrm{GK}$. Obviously the mean collision frequency can now be found by averaging $\nu_{\gamma \gamma}(k)$. The mean free path of the photon in the black-body bath is then

$$
l=\frac{c}{\text { mean collision frequency }}
$$

and according to (Partovi, 1994) it is: 


$$
l[m]=9 \times 10^{-6}\left(\frac{T[K]}{5.9 \times 10^{9}}\right)^{-9} .
$$

The probability that the photon exchange between two charges in the distance $r$ will be destroyed by the photon-photon interaction is obviously $r / l$. It is evident that if the distance between both charges is $r \geq l$, then there is no interaction between these charges because every exchanged photon interacts with some of the sea photons. So, $V_{T}$ is equal to zero with probability $r / l$ and it is equal to $V(r)$ with probability $1-r / l$ as a consequence of the existence of the mean free path of photons. Thus we can easily write the two-body potential in the photon sea in of the form

$$
V_{T}(r) \approx\left(1-\frac{r}{l}\right) V(r), \quad r \leq l,
$$

and $V_{T}=0$ for $r>l$.

The dependence of the coupling constant on temperature follows from the last formula. Namely, if we put at zero temperature

$$
V(r)=-\frac{a}{r}
$$

then it is obvious that

$$
a(r, T) \approx\left(1-\frac{r}{l}\right) a(0)
$$

and $a(r, T)=0$ for $r \geq l$, where the mean free path $l$ depends on temperature.

We see that the coupling constant following from our definition of the potential at finite temperature depends not only on temperature, but also on the distance between the particles. So we have got the specific form of the running coupling constant. It is easy to see that for sufficiently large temperature $T$ the coupling constant is approximately zero and the interaction of two particles in the photon medium is also approximately zero. So, particles are free in the sufficient high temperature regime.

While the mean free path for hydrogen under standard conditions is about $10^{-6} \mathrm{~m}$, allowing hydrogen to behave like a continuous medium on scales larger than a micron, the same quantity for a photon gas is a staggering $10^{61} \mathrm{~m}$, implying that under ordinary conditions photons behave like a collection of independent, free-streaming particles on any physically meaningful scale (Partovi, 1994). It means that the influence of the photonphoton interaction on the hydrogen spectrum is practically beyond any experimental possibilities.

The influence of the black-body radiation on the energy shift was determined experimentally by Hollberg and Hall (1984). Following the detailed calculation of Farley and Wing (1981), and using the high-precision laser spectroscopic techniques, they have measured the predicted shift of Rydberg energy levels induced by black-body radiation. The 
implementation of laser precision measurement and optical heterodyne techniques has allowed the measurement of atomic energy shifts with a precision not possible before. These measurements are consistent with the predicted finite-temperature radiative corrections to atomic energy levels induced by black-body radiation, not, of course, as a result of the photon-photon interaction, but as a result of the dynamical Stark shift and the electricdipole depopulation rate, which are effects generated by the black-body radiation and not by the change of interaction potential in the photon sea.

\section{Discussion}

We have seen how the finite-temperature potential follows from the temperature Green function in the framework of the Schwinger field theory. We have concentrated only on the Coulomb and Yukawa potentials and we derived the finite-temperature terms to these potentials using the Green fuctions of a photon with and without radiative corrections, and then by considering the processes expressed by the Feynman diagrams.

Baier and Kalashnikov (1994) determined the so-called non-Abelian screening potential in the situation beyond leading order. It is evident that the screening potential corresponds to a different physical situation than that considered by us. They determined the static QCD colour-singlet potential beyond the leading order exploiting the infra-red limit of the time-time component of the gluon self-energy $\Pi_{44}(0, \mathbf{k})$. They worked in the temporal axial gauge and used the full Schwinger-Dyson equation for $\Pi_{44}$, which includes the ring graphs and non-perturbative vertex corrections. Gauge covariance was guaranteed by the exact Slavnov-Taylor identities in order to construct the three-gluon vertex. Their result for the screening static potential $V(r)$ is as follows:

$$
V(r)=-\frac{N^{2}-1}{2 N} g^{2}(T) \int \frac{d^{3} q}{(2 \pi)^{3}} \frac{e^{i \mathbf{q} \mathbf{r}}}{q^{2}+\Pi_{44}(0, q)},
$$

where $r=|\mathbf{r}|, q=|\mathbf{q}|$ and $g(T)$ is the coupling constant at finite temperature.

The final result of their calculations is the formula:

$$
\left.V(r)\right|_{m r \longrightarrow \infty} \approx \frac{N^{2}-1}{8 \pi^{2}} \frac{3 g^{2}(T)}{(r m)^{6}} T .
$$

It is evident that the photon-photon interaction forms the analogue of the phononphonon interaction in solid states and condensed matter. Thus, in analogy with photons, we can define the mean free path of phonons and expect in the case of solid states and condensed matter the mean free path of phonons to be as follows:

$$
l=\frac{A}{T^{m}},
$$

where $A$ and $m$ are constants corresponding to the specific matter and $T$ is the temperature. It is well known that phonons are the fundamental medium which makes possible the 
heat transfer in solid states, and according to Rietschel (1994) electron-phonon interaction is the relevant mechanism in the high-temperature superconductivity.

The mean free path (46), although derived only by analogy with the photon situation in QED, is in fact realistic, because according to Anderson (1981) there exist so-called non-resonant phonon scattering mechanisms in a solid characterized by a mean free path $l \approx T^{-3}$ which is in agreement with the analogous formula (46). So, the relevance of the mean free path in solid state physics is obvious, as shown also in the article by Yang et al. (1994), where it is demonstrated that the mean free path of phonons can be determined experimentally by measuring thermal diffusivity without having the need to measure the thermal conductivity and the heat capacity per unit volume.

The mean free path plays a fundamental role in particle physics, as was shown for instance by Haglin and Pratt (1994), who studied mean free paths of pions and kaons using relativistic kinetic theory at temperature and densities characteristic of hadronic matter.

\section{References}

Anderson, A. C. (1981). Amorphous Solids: Low Temperature properties, ed. W. A. Phillips Berlin: Springer p. 65.

Baier, R. and Kalashnikov, O. K. (1994). The nonabelian screening potential beyond the leading order, Phys. Lett. B 328, 450.

Bernard, C. W. (1974). Feynman rules for gauge theories at finite temperature, Phys. Rev. D 9, 3312.

Cox, P. H., Hellman, W. S. and Yildiz, A. (1984). Finite temperature corrections to field theory: Electron mass and magnetic moment, and vacuum energy,

Ann. Phys. (N.Y.) 154, 211.

Dittrich, W. (1978). Source Methods in Quantum Field Theory, Fortschritte der Physik 26, 289.

Dolan, L., Jackiw, R. (1974). Symmetry behavior at finite temperature, Phys. Rev. D 9, 3320.

Donoghue, J. F., Holstein, B. R. and Robinett, R. W. (1985). Quantum Electrodynamics at Finite Temperature, Ann. Phys. (NY) 164 No. 2, 233.

Farley, J. W. and Wing, W. H. (1981). Accurate calculation of dynamic Stark shifts and depopulation rates of Rydberg energy levels induced by blackbody radiation. Hydrogen, helium, and alkali-metal atoms, Phys. Rev. A 23, 2397. Haglin, K. and Pratt, S. (1994). On the mean free paths of pions and kaons in hot hadronic matter, Phys. Lett. B 328, 255. nucl-th/9404007v1 6 Apr 1994 
Hollberg, L. and Hall, J. L. (1984). Measurement of the Shift of Rydberg Energy Levels Induced by Blackbody Radiation, Phys. Rev. Lett. 53, 230.

Johansson, A. E., Peressutti, G. and Skagerstam, B. S. (1986). Quantum field theory at finite temperature, Renormalization and radiative Nucl. Phys. B 278, 324.

Kajantie, K. and Kapusta, J. (1985). Behaviour of gluons at high temperature, Ann. Phys. (N.Y.) 160, 477.

Pardy, M. (1989). Finite-temperature Čerenkov radiation, Phys. Lett. A 134 No. 6, 357. Pardy, M. (1994a). The two-body potential at finite temperature, CERN-TH.7397/94

Pardy M. (1994b). The Čerenkov effect with radiative corrections, Phys. Lett. B 325, 517.

Pardy, M. (1994c). The synchrotron production of photons with radiative corrections, Phys. Lett. A 189, 227.

Partovi, M. Hossein. (1994). QED corrections to Plancks radiation law and photon thermodynamics, Phys. Rev. D $50,1118$.

Rietschel, H. (1994). Relevance of electron-phonon coupling in high- $T_{c}$ superconductors, Journal of Low Temperature Physics, 95 Nos 1/2, 293.

Schwinger, J. Particles, Sources and Fields, Vol. I,

Addison-Wesley, Reading, Mass., (1970).

Schwinger, J., Tsai, W. Y. and Erber, T. (1976). Classical and Quantum

Theory of Synergic Synchrotron-Čerenkov Radiation, Ann. Phys. (NY) 96 No. 2, 303.

Schwinger, J. Particles, Sources and Fields Vol. II,

Addison-Wesley, Reading, Mass., (1973).

Tryon, E. P. (1974). Quantum Electrodynamics in a Photon Sea,

Phys. Rev. Lett. 32, 1139.

Yang, G., Migone, A. D. and Johnson, K. W. (1994). Relationship between thermal diffusivity and mean free path, Am. J. Phys. 62 (4), 370.

Weinberg, S. (1974). Gauge and global symmetries at high temperature, Phys. Rev. D 9, 3357.

Weldon, H. A. (1994). Cancellation of infrared divergences in thermal QED, Nucl. Phys. A 566, 581c.

Zhukovskii, V. Ch., Midodashivili, P. G. and Éminov, P. A. (1995).

Imaginary part of the electron mass operator in a static uniform magnetic field at finite temperature and nonzero chemical potential, JETP 80, No. 2, 356. 